patients were included as having active DU or pulmonary arterial hypertension who had active DU in the last 3 months. A DU was defined as an area with visually discernable depth and a loss of continuity of epithelial coverage, which could be denuded or covered by a scab or necrotic tissue. If the area was denuded, the ulcer was designated active. If denudation could not be judged because of the presence of overlying scab or necrotic tissue, ulcers presenting with features, including underlying pain, based on investigator clinical judgment to be consistent with loss of epithelialization, epidermis, or dermis, and requiring treatment, were designated as active. The effectiveness and tolerability of bosentan was monitored within 24 weeks of study treatment, while healing of DU, largest diameter and the new incidence were also assessed up to week 24

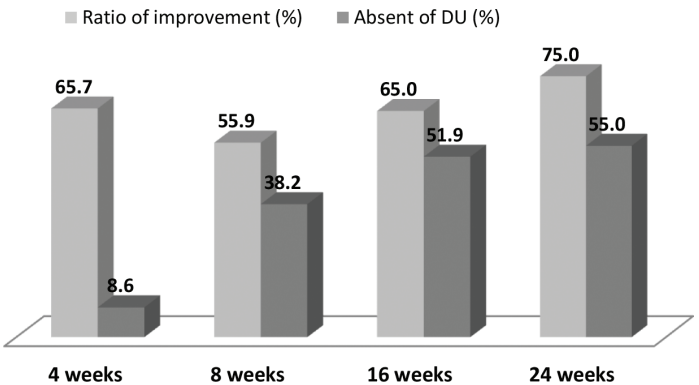

Serial change of DU

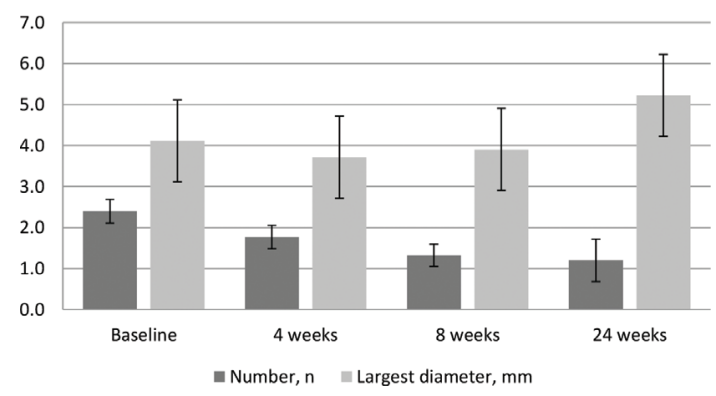

Results: The primary objective of this study was to assess the efficacy of bosentan in reducing DU including the complete healing rate. Secondary endpoints included development of new DU, assessment of discontinuation, measures of nailfold capillaroscopic (NFC) change. Thirty-five SSc patients were enrolled and seventeen patients were included in the NFC analysis. Mean age was 50.5 year-old, $85.7 \%$ was woman, $68.6 \%$ were diffuse type of SSc, mean mRSS was 15.4 and mean disease duration was 77.9 months. Among 35 patients, 20 patients was finished 24 weeks treatment duration. The main reason for discontinuation is when the patient wants to stop because of the full effect of the drug $(n=12,80 \%)$. The side effects of the drug occurred in 4 cases during the treatment period. The mean number of DUs before treatment was 2.4 and was statistically decreased to $1.2(p=0.001)$. From the 4 th week of bosentan treatment, improvement of DU number was observed in $65.7 \%$ and complete healing was observed in $55 \%$ at 24 weeks. However, the mean size of largest DU diameter, remained at the end of treatment, was slightly increased from 4.1 to $5.2 \mathrm{~mm}$, with no statistical significance. There was no statistically significant difference in the semiquantitative changes of NFC before and after bosentan treatment.

Conclusion: Administration of bosentan over 24 weeks was statistically associated with complete healing and significant reduction in net number of DU in Korean patients with SSc. However, the size of the remaining DU, that did not respond to bosentan, has rather increased.

Disclosure of Interests: None declared

DOI: 10.1136/annrheumdis-2019-eular.7157

\section{AB0670 \\ INTESTINAL ANGIODISPLASIAS IN THE PATIENT WITH SYSTEMIC SCLEROSIS}

Carolina Merino Argumánez ${ }^{1}$, Olga Rusinovich ${ }^{1}$, María Espinosa ${ }^{2}$, Consuelo Ramos Giráldez ${ }^{3}$, Carmen Barbadillo Mateos ${ }^{1}$, José Luis Andréu Sánchez ${ }^{1}$, Hilda Godoy ${ }^{1} .{ }^{1}$ Hospital Puerta de Hierro, Majadahonda (Madrid), Spain; ${ }^{2}$ Hospital Infanta Sofía, Madrid, Spain; ${ }^{3}$ Valme Hospital, Sevilla, Spain

Background: Gastric angiodysplasia and the typical "watermelon stomach" aspect is one of the most characteristic vascular manifestations in systemic sclerosis (SS). These angiodysplasias can affect the intestine ocasionally as well as the stomach.

Objectives: Description of a series of 5 patients with systemic sclerosis and presence of intestinal angiodysplasia (IA).

Methods: Retrospective unicentric observational study in a tertiary hospital. Demographic data, comorbidities, manifestations of the disease, serological profile, concomitant treatments and bleeding manifestations, as well as endoscopic findings and received treatment were collected. Descriptive statistics was used to present the data.

Results: We reviewed the medical records of the 88 patients with a diag nosis of SS followed up in the Rheumatology Service. Five cases $(5.6 \%)$ of IA were identified (4 women and one male), with a mean age of 73 years (SD 13.71). In one case, the diagnosis of SS and IA was simultaneous. In the remaining 4 cases, the mean time from the diagnosis of ES to the diagnosis of the IA was 7.75 years (SD 2.6). Regarding the clinical presentation, 4 of the cases presented as anemia with iron deficiency. In these cases IA were found in the endoscopic studies. In one case, IA were a finding in the context of the study of diarrhea. In 4 of the cases, the IA were in the small intestine, so the diagnosis was made using an endoscopic capsule. In one case they were found in the colon, so the diagnosis was by colonoscopy. Only one of the cases of angiodysplasias in the small intestine required specific treatment with argon laser by enteroscopy. One case required a transfusion of packed red blood cells. In the remaining cases, treatment with oral or intravenous iron therapy was enough. In all 5 cases, it was a limited form of the SS with the presence of anticentromere antibodies. All cases presented Raynaud's phenomenon, 3 of them with digital ulcers. Three of the cases associated primary biliary cholangitis, 3 cases pulmonary hypertension, 3 cases peristaltic involvement, 1 case interstitial lung disease and 1 case restrictive cardiomyopathy. As comorbidities, we identified 1 case of essential mixed cryoglobulinemia associated with hepatitis $\mathrm{C}$ virus, 2 cases of valvular heart disease, 2 cases of atrial fibrillation. Conclusion: The extragastric localization of the angiodysplasias should be taken into account in the patient with SS and iron deficiency, especially in those with a limited form of the illness and positive anticentromere antibodies

Disclosure of Interests: Carolina Merino Argumánez: None declared, Olga Rusinovich: None declared, María Espinosa: None declared, Consuelo Ramos Giráldez Speakers bureau: Sanofi, Carmen Barbadillo Mateos: None declared, José Luis Andréu Sánchez: None declared, Hilda Godoy: None declared

DOI: 10.1136/annrheumdis-2019-eular.7944

\section{AB0671 DISCORDANCE IN ANTI-TIF-1 $\gamma$ ANTIBODIES DETECTION THROUGH COMMERCIAL KIT IN COMPARISON WITH HOMEMADE IMMUNOBLOT: CLINICAL EVALUATION}

Ana Milena Millán Arciniegas ${ }^{1}$, M. Angeles Martinez ${ }^{2}$, Anais Mariscal ${ }^{2}$, Andres Baucells ${ }^{2}$,Leticia Alserawan², Ernesto Trallero ${ }^{3}$,Cesar Díaz-Torné1, Ana Laiz ${ }^{1}$, Berta Magallares ${ }^{1}$, Patricia Moya ${ }^{1}$, Hyesang Park ${ }^{1}$, Andrea GarcíaGuillén $^{1}$, Sycille Jeria ${ }^{1}$, David Lobo ${ }^{1}$,Albert Gil ${ }^{4}$, Coral Gozalvez ${ }^{1}$, Susana

P. Fernandez-Sanchez ${ }^{1}$,Laura Martínez-Martínez ${ }^{2}$, Esther Moga ${ }^{2}$,

Hector Corominas ${ }^{1}$, Cándido Juarez ${ }^{2}$, Ivan Castellví ${ }^{1}$, Albert Selva-O'callaghan ${ }^{4}$.

${ }^{1}$ Rheumatology Unit, Hospital de la Santa Creu i Sant Pau, Barcelona, Spain;

${ }^{2}$ Immunology Department, Hospital de la Santa Creu i Sant Pau, Barcelona, Spain;

${ }^{3}$ Rheumatology Unit, Vall d'Hebron General Hospital, Barcelona, Spain; ${ }^{4}$ Systemic Autoimmune Diseases Unit, Vall D'Hebron General Hospital, Internal Medicine Department, Barcelona, Spain

Background: The antibody against the transcriptional intermediate facto $1 \gamma$ (Anti-TIF1 $\gamma$ or Anti-p155), is considered specific for dermatomyositis (DM) and its a marker of cancer risk. Our center carries out the determination of Anti-TIF1 $\gamma$ by a homemade immunoblot (IB) technique with a $155 \mathrm{kDa}$ TIF1 $\gamma$ human recombinant protein (OriGene). Currently there is 
a commercial technique of IB to detect TIF1 $\gamma$ (Euroimmun), however, commercial kits sometimes elaborate antigens without using whole protein structure. To date, there are no studies that study the agreement between the commercial kit and homemades IB.

Objectives: 1-To compare the commercial IB results of Anti-TIF1 $\gamma$ with homemade IB.

2-To describe and compare the clinical characteristics of the patients analyzed by both techniques.

METHODS: Observational retrospective study that included adult patients with some determination of Anti-TIF1 $\gamma$ antibodies since 2014 in two tertiary-level university hospitals. We collected demographic and clinical data of all patients. Taking into account their diagnoses we grouped them into: Cancer Associated Myositis (CAM), DM without cancer, Systemic Autoimmune Diseases (SAD) non-DM and other diagnoses. Subsequently, the clinical agreement with the IB results of Anti-TIF1 $\gamma$ performed by commercial (clB) and homemade (hIB) technique were analyzed. A p value $<0.05$ was considered statistically significant.

RESULTS: 48 patients were recruited (77.1\% women). 13 had diagnosis of CAM, 19 of DM without cancer, 7 of other non-DM EAS, and 9 had other diagnoses.

Of all DM (CAM and DM without cancer), 12 were positive for both techniques. 5 presented discordant results $(4 \mathrm{hIB}$ positive with negative clB and $1 \mathrm{clB}$ positive with negative hIB). 15 DM were negative for the two techniques, of which $8 / 15$ had other antibodies associated with Autoimmune Myopathy (3 NXP-2, 1 Mi2, 1 MDA5, 1 SAE, 1 Jo1 and 1 Ro52). When we analyzed patients with CAM with both techniques, 10/13 presented positive hIB vs 8/13 clB positive. DM without cancer, 6/19 had positive hIB vs 5/19 clB positive. The sensitivity and specificity of each test was evaluated in CAM and DM without cancer, being $61.5 \%$ and $73.7 \%$ respectively for clB $(p=0.071)$, compared to $76.9 \%$ and $68.4 \%$ for hIB ( $p=0.029$ ).

In non-DM SAD group, 1 patient had positive determination for both techniques, 3 had positive clB with negative hIB, and $3 \mathrm{clB}$ negative with positive hIB. We also found other antibodies in 6/7 patients with non-DM SAD. The 9 patients with other diagnoses were: 1 positive for both techniques and 8 positive for $\mathrm{ClB}$ and negative for hIB.

CONCLUSIONS: Homemade IB with $155 \mathrm{kDa}$ recombinant protein was superior to clB to detect TIF $\gamma$ in our serie of patients with CAM. This could have clinical implications in screening and early detection of neoplasia that affect false negative by clB.

A positive $\mathrm{clB}$ in patients not suspected as a SAD were negative for hIB in the major part of cases.

It would be recommended to check the commercial IB using homemade IB, due to its known clinical-therapeutic implications.

Disclosure of Interests: Ana Milena Millán Arciniegas: None declared, M. ANGELES MARTINEZ: None declared, ANAIS MARISCAL: None declared, ANDRES BAUCELLS: None declared, Leticia Alserawan: None declared, Ernesto Trallero: None declared, Cesar Díaz-Torné: None declared, Ana Laiz Consultant for: Lilly, Novartis, AbbVvie, MSD, UCB and Janssen, Speakers bureau: Lilly, Novartis, Abvvie, MSD, UCB and Janssen, Berta Magallares: None declared, Patricia Moya: None declared, HyeSang Park: None declared, Andrea García-Guillén: None declared, Sycille Jeria: None declared, DAVID LOBO: None declared, Albert Gil: None declared, Coral Gozalvez: None declared, Susana P. Fernandez-Sanchez: None declared, Laura Martínez-Martínez: None declared, Esther Moga: None declared, Hector Corominas: None declared, Cándido Juarez: None declared, Ivan Castellví Consultant for: I received fees less than 5000USD as a consultant for Kern and Actelion, Paid instructor for: I received fees less than 2000USD as a instructor for Boehringer -Ingelheim, Novartis and Gebro, Speakers bureau: ND, Albert Selva-O'Callaghan: None declared DOI: 10.1136/annrheumdis-2019-eular.6954

\section{AB0672 RENAL INVOLVEMENT IN PATIENTS WITH DERMATOMYOSITIS AND POLYMYOSITIS}

Simeon Monov ${ }^{1}$, Daniela Monova ${ }^{2}$, Russka Shumnalieva $^{1}$, Elena Miloshova ${ }^{2}$.

${ }^{1}$ Medical University - Sofia, Department of Rheumatology, Sofia, Bulgaria;

${ }^{2}$ Medical Institute, Medical University - Sofia, Department of Internal Diseases, Sofia, Bulgaria

Background: Dermatomyositis (DM) and polymyositis (PM) are characterized by moderate to severe muscle weakness and inflammatory lesions in the muscle. They lead to frequent, and, in some cases, life-threatening extramuscular (lung, cardiac, neurologic, kidney) complications.

Objectives: The aim of this study was to determine the incidence, the severity, the course and outcome of renal diseases in patients with DM or PM.
Methods: We identified 84 patients (68 female, 16 male) with the diagnosis of DM and 23 (14 female, 9 male) with PM. Diagnosis of DM and PM was based on the ENMC classification. The mean follow-up was 5,6 $\pm 3,8$ years. Evaluation of extramuscular involvement was mainly performed in the presence of suggestive clinical signs. Autoantibodies were detected by ELISA. Acute kidney injury (AKI) was defined as an acute doubling of serum creatinine level. Chronic kidney disease (CKD) was defined if an estimated glomerular filtration rate (eGFR) $<60 \mathrm{~mL} / \mathrm{min}$ on at least 2 measurements 3 months apart.

Results: Of the 107 patients, 26 were found to have suffered varying degree of renal involvement. All the 26 patients $(22$ patients with DM and 4 with PM) had varying degree of proteinuria and haematuria. Renal involvement consisted of $\mathrm{AKI}$ in 8 patients and $\mathrm{CKD}$ in 18 patients. Among patients with $\mathrm{AKI}, 6(75 \%)$ progressed to $\mathrm{CKD}$, including 2 patients who reached end-stage renal disease (ESRD). Acute tubular necrosis with renal failure developed in 3 patients with $P M$ and in 4 patients with DM. In descriptive analysis, male sex, cardiovascular risk factors, the severity of muscle injury, heart involvement, and initial proteinuria $(>0.3 \mathrm{~g} / \mathrm{d})$ were associated with AKI. In multivariate survival analysis, only age at disease onset, male sex, history of cardiovascular events, and a previous episode of AKI were identified as risks factors of CKD. Although the proteinuria and haematuria responded to therapy (except in 2 cases), the creatinine clearance remained decreased during the treatment. Kidney biopsy was performed in 16 patients and showed chronic tubulointerstitial nephritis ( 7 cases), minimal change disease (2 cases), focal segmental glomerulosclerosis lesions (3 cases), IgA nephropathy ( 2 cases), membranous nephropathy (1 case), and amyloid deposits (1 case).

Conclusion: Our data clearly indicate that DM and PM are associated with a wide range of renal disorders. Renal damage was noted in $24,3 \%$ of all included patients. AKI occurred in 7,48\% of cases and CKD developed in $16,82 \%$ of the patients. AKI and CKD contribute to an increased morbidity in these patients. AKI and CKD are probably not a direct manifestation of PM or DM, but rather result from drug and myoglobin-induced renal damage. However, $\mathrm{AKI}$ and $\mathrm{CKD}$ contribute to increased morbidity in these patients

Disclosure of Interests: None declared

DOI: 10.1136/annrheumdis-2019-eular.1525

\section{AB0673 CHARACTERISTICS OF THE CAPILLAROSCOPY PATTERN IN SYSTEMIC AUTOIMMUNES DISEASES}

Atusa movasat Hajhkan, Ana Isabel Turrión, Henry Moruno, Cristina Bohórquez, Ana Pérez Gómez, Eduardo Cuende, Fernando Albarrán, Ana Sánchez Atrio, Lucía Ruiz, Paula Pretel, Laura Barrio Nogal, Valentina Emperiale, Adrián Abbasi, Melchor Álvarez de Mon. hospital principe de asturias, alcala de henares, madrid, Spain

Background: Nailfold capillaroscopy (CP) is a simple, safe, low cost and non-invasive screenig test and together with the study of autoantibodies, is useful in the diagnosis of systemic sclerosis (SSc). It demonstrates certain specific patterns which are characterized by the presence of dilated capillaries, haemorrhages and avascular areas. Other systemic autoimmune diseases (SAD) do not have a specific pattern, but certain alterations in the morphology of the capillaries such as increased tortuosity have been described in SLE. In other diseases such as mixed connective tissue disease (MCTD), inflammatory myopathies and overlap syndromes, findings may be different to the pathological findings consis tent with scleroderma pattern.

Objectives: Description of nailfold capillaroscopic findings in different SAD. Methods: The study included 255 patients referred for $\mathrm{CP}$ that was performed in 8 fingers, and always by the same observer. The following findings were considered as "scleroderma pattern": Local or global capillary loss (> 20\%), haemorrhages: two or more in at least two fingers and enlarged capillaries: two or more capillary with double caliber or more in at least two different fingers. Abundant evidence of tortuosity (> $20 \%$ ) as ringlets tangles and no evidence of disorders described above was considered nonspecific, tortuous pattern or suspects SAD. Statistical analysis was performed with SPSS 19.0 program.

Results: CP was performed in 255 patients, 31 males (12\%) and 224 $(88 \%)$ women. 23 patients $(9 \%)$ had a diagnosis of SSc, 6 patients $(2 \%)$ of SLE, other $6(2 \%)$ of Sjögren's syndrome, 7 (3\%) of MCTD, $4(1.5 \%)$ of inflammatory myopathy and $2(0.8 \%)$ of overlap syndrome. 18 patients (7\%) had positive ANA and Raynaud without another data suggestive of SAD, 76 patinents with primary Raynaud (30\%) and 66 patients (26\%) with a miscellany of other diseases such as antiphospholipid syndrome, vasculitis, fibromyalgia and psoriatic arthritis. Also, 29 patients (12.4\%) were sent for performing CP for suspected systemic sclerosis and 16 